\title{
HAUSDORFF DIMENSION AND EXCEPTIONAL SETS OF LINEAR TRANSFORMATIONS
}

\author{
R. KAUFMAN and P. MATTILA
}

1. Introduction. Let $m$ and $n$ be positive integers with $m \leq n$ and let $O^{*}(n, m)$ be the set of all orthogonal projections of $R^{n}$ onto $R^{m}$ (see $[3,1.7 .4])$. A linear mapping $p: R^{n} \rightarrow R^{m}$ belongs to $O^{*}(n, m)$ if and only if $p$ maps the orthogonal complement of $\operatorname{ker} p$ isometrically onto $R^{m}$. Considered as a subset of the space of all linear mappings of $R^{n}$ into $R^{m}$, which is denoted by $\operatorname{Hom}\left(R^{n}, R^{m}\right)$ and identified with $R^{n m}, O^{*}(n, m)$ has positive and finite $m(2 n-m-1) / 2$-dimensional Hausdorff measure (see $[3,3.2 .28(5)])$. It was proved first in [5] for $m=1, n=2$ and then in [8] for general $m, n$ that if $E$ is a Borel (or, more generally, Suslin) set in $R^{n}$ with $\operatorname{dim} E=s \leq m$, then $\operatorname{dim} p(E)=s$ for $\mathscr{H}^{s+m(2 n-m-3) / 2}$ almost all $p \in O^{*}(n, m)$. Here dim means Hausdorff dimension and $\mathscr{H}^{t}$ is the $t$-dimensional Hausdorff measure. In [6] an example of a compact plane set was given such that the corresponding exceptional set of projections has positive Hausdorff dimension. In this paper we shall show that the number $s+m(2 n-m-3) / 2$ is the best possible upper bound for the Hausdorff dimension of the exceptional set. We shall also indicate how the result mentioned above can be generalized to a larger class of sets of linear transformations.

2. Preliminaries. Besides what is presented in the introduction, we shall use the following notation: The orthogonal group of $R^{n}$ is denoted by $O(n)$. The Grassmann manifold of all $m$-dimensional linear subspaces of $R^{n}$ is $G(n, m)$. For $V \in G(n, m), P_{V}$ is the orthogonal projection of $R^{n}$ onto $V$ and $V^{\perp}$ is the orthogonal complement of $V$. If $f \in \operatorname{Hom}\left(R^{n}, R^{m}\right)$, then

$$
\begin{gathered}
\|f\|=\max \left\{|f(x)|: x \in S^{n-1}\right\}, \\
l(f)=\min \left\{|f(x)|: x \in S^{n-1} \cap(\operatorname{ker} f)^{\perp}\right\}
\end{gathered}
$$

and $r(f)$ is the rank of $f$. 
There exists a positive number $c$ depending only on $m$ with the following properties:

2.1. If $V, W \in G(n, m)$ and $\left|w-P_{V}(w)\right| \leq \delta|w|$ for $w \in W$, then $\left\|P_{V}-P_{W}\right\| \leq c \delta$.

2.2. If $f, g \in \operatorname{Hom}\left(R^{n}, R^{m}\right)$ and $r(f)=r(g)$, then

$$
\begin{gathered}
l(f)\left|x-P_{\text {ker } f}(x)\right| \leq|f(x)| \text { for } x \in R^{n}, \\
l(f)\left\|P_{\text {ker } f}-P_{\text {ker } g}\right\| \leq c\|f-g\| .
\end{gathered}
$$

One can prove 2.1 by choosing an orthonormal base $\left\{w_{1}, \ldots, w_{m}\right\}$ for $W$ and by constructing an orthonormal base $\left\{v_{1}, \ldots, v_{m}\right\}$ for $V$ such that $\left|v_{i}-w_{i}\right| \leq c^{\prime} \delta$ where $c^{\prime}$ depends only on $m$. The first inequality in 2.2 is an immediate consequence of the definition of $l(f)$, and the second follows from the first and 2.1.

The metric $d$ and Hausdorff measures on $G(n, m)$ are defined via the identification used in [3, 3.2.28]. The inequalities [9, Chapter I, 12 (17), $15(7)]$ and 2.1 imply that there is a positive number $b$ such that

$$
b^{-1} d(V, W) \leq\left\|P_{V}-P_{W}\right\| \leq b d(V, W)
$$

for $V, W \in G(n, m)$. Therefore, for the purposes of this paper, we could as well identify $V \in G(n, m)$ with $P_{V}$.

3. Proposition. Let $k$ and $n$ be positive integers and let $M$ be a Borel set in Hom $\left(R^{n}, R^{k}\right)$. For $x \in S^{n-1}$ denote

$$
M_{x}=\{f \in M: f(x)=0\} .
$$

Suppose that there exist real numbers $c>0, C>0$ and $v \geq 0$ and that to each $g \in O(n)$ corresponds a mapping $A_{g}: M \rightarrow M$ such that the following conditions are satisfied for all $f \in M$ and $g \in O(n)$ :

(1) $\operatorname{ker} f \circ g \subset \operatorname{ker} A_{g} f$,

(2) $\left\|f-A_{g} f\right\| \leq C\|f\|\left\|\mathbf{1}_{R^{n}}-g\right\|$, where $\mathbf{1}_{R^{n}}$ is the identity mapping of $R^{n}$,

(3) $\mathscr{H}^{v}\left(M_{x}\right) \leq C$ for all $x \in S^{n-1}$,

(4) $\mathscr{H}^{v}\left\{h^{\prime} \in M_{x}:\left\|h^{\prime}-h\right\| \leq r\right\} \geq c r^{v}$ for all $x \in S^{n-1}, \quad h \in M_{x}$ and $0<r<1$.

If $E$ is a Suslin set in $R^{n}$ and $\operatorname{dim} E=s$, then $\operatorname{dim} f(E)=s$ for $\mathscr{H}^{s+\nu}$ almost all $f \in M$.

Since $\operatorname{dim} f(E)=s$, if $\operatorname{ker} f=\varnothing$, we may assume that $\operatorname{ker} f \neq \varnothing$ for $f \in M$. Further, since

$$
M \backslash\{0\}=\bigcup_{j=1}^{\infty} N_{j} \text {, where } N_{j}=\{f \in M: l(f)>1 / j,\|f\|<j\},
$$


it is sufficient to prove that $\operatorname{dim} f(E)=s$ for $\mathscr{H}^{s+v}$ almost all $f \in N_{j}$ for all $j$. The proof of this fact is almost identical with that of Theorem $5.6(\mathrm{~b})$ in [8]. Indeed, after fixing $j$ we only have to verify the following analogue of the formula (1) occurring in the proof of Lemma 5.5 in [8]:

There exists a positive number $c_{1}$ such that if $f \in N_{j}, x \in S^{n-1}$, $\delta>0$ and $|f(x)| \leq \delta$, then $\|f-h\| \leq c_{1} \delta$ for some $h \in M_{x}$.

To see this, take $z \in S^{n-1} \cap \operatorname{ker} f$ such that $\left|P_{\text {ker } \mathrm{f}}(x)\right| z=P_{\text {ker } \mathrm{f}}(x)$. Then $|x-z| \leqq 2 j \delta$ by 2.2. Choose $g \in O(n)$ such that $g(x)=z$ and $\left\|\mathbf{1}_{R^{n}}-g\right\|=|x-z|$. Then $f(g(x))=0, \quad A_{g} f \in M_{x}$ by (1), and $\left\|f-A_{g} f\right\| \leq c_{1} \delta$ with $c_{1}=2 j^{2} C$ by $(2)$.

4. Remarks. One can often apply this proposition in the following situation: To each pair of positive integers $m, n, m \leq n$, corresponds a smooth submanifold $M(n, m)$ of some space Hom $\left(R^{n}, R^{k}\right)$ such that $M(n, m)_{x}$ is isometric with $M(n-1, m)$ for $m<n$ and $x \in S^{n-1}$. Then the conditions (3) and (4) are satisfied with $v=\operatorname{dim} M(n-1, m)$ if $\mathscr{H}^{\nu}(M(n-1, m))<\infty$ and $M(n-1, m)$ is "sufficiently homogeneous". Such is the case if $M(n, m)$ is $O^{*}(n, m)$ or $\left\{P_{V}: V \in G(n, m)\right\}$. In the first case one can define $A_{g}$ by $A_{g} p=p \circ g$ and in the second by $A_{g} P_{V}=P_{\mathrm{g}^{-1}(V)}$. Then all the assumptions are in force. If $M(n, m)=$ Hom $\left(R^{n}, R^{m}\right)$, then $(3)$ is false. However the proposition is true even in this case, because all the assumptions hold with $A_{g} f=f \circ g$ for $M=\left\{f \in \operatorname{Hom}\left(R^{n}, R^{m}\right):\|f\|<R\right\}$ whenever $0<R<\infty$.

The dimensions of the manifolds $O^{*}(n, m), G(n, m)$ and Hom $\left(R^{n}, R^{m}\right)$ are $m(2 n-m-1) / 2, m(n-m)$ and $m n$ (see $[3,3.2 .28(5)])$, so that the upper bounds for the dimensions of the exceptional sets are $s+m(2 n-m-3) / 2, s+m(n-m-1)$ and $s+m(n-1)$, respectively. We shall next show that these upper bounds may be attained. More precisely, we shall prove

5. The or e m. If $m$ and $n$ are integers and $s$ is a real number with $0<s \leq m<n$, then there exists a compact set $E \subset R^{n}$ such that $\operatorname{dim} E=s$ and

$\operatorname{dim}\left\{p \in O^{*}(n, m): \operatorname{dim} p(E)<s\right\}=s+m(2 n-m-3) / 2$,

$\operatorname{dim}\left\{V \in G(n, m): \operatorname{dim} P_{V}(E)<s\right\}=s+m(n-m-1)$,

$\operatorname{dim}\left\{f \in \operatorname{Hom}\left(R^{n}, R^{m}\right): r(f)=m, \operatorname{dim} f(E)<s\right\}=s+m(n-1)$.

The following lemma is an immediate consequence of $[3,2.10 .25]$ :

6. L e m ma. If $X$ and $Y$ are metric spaces, $Y$ is o-compact, $F: X \rightarrow Y$ is Lipschitzian, $\varnothing \neq B \subset Y, 0 \leq v<\infty$ and $\mathscr{H}^{v}\left(F^{-1}\{y\}\right)>0$ for $y \in B$, then $\operatorname{dim} F^{-1}(B) \geq \operatorname{dim} B+v$. 
Proof. We may assume that $Y$ is compact. If $t \geq 0$, it follows from $[3,2.10 .25]$ that for a positive number $c$

$$
\int_{B}^{*} \mathscr{H}^{\nu}\left(F^{-1}\{y\}\right) d \mathscr{H}^{t} y \leq c \mathscr{H}^{t+v}\left(F^{-1}(B)\right) .
$$

Hence $\mathscr{H}^{t+v}\left(F^{-1}(B)\right)=0$ implies $\mathscr{H}^{t}(B)=0$, which means that $\operatorname{dim} F^{-1}(B) \geq \operatorname{dim} B+v$.

7. Proof of Theorem 5. We begin with the case of $G(n, m)$ and assume first that $m=n-1$. Let $\left(N_{k}\right)$ be a strictly and rapidly increasing sequence of positive integers, e.g. $N_{k+1}>N_{k}^{k}$, and let $E$ be the set of all $\left(x_{1}, \ldots, x_{n}\right) \in R^{n}$ such that

$$
-1 \leq x_{j} \leq 1, \quad\left\|N_{k} x_{j}\right\| \leq N_{k}^{1-n / s} \quad \text { for } 1 \leq j \leq n, k \geq 1 .
$$

(For a real number $t,\|t\|$ is the distance from $t$ to the nearest integer.) Then $\operatorname{dim} E=s$. This follows from [2, Theorem 10]. The method goes back to Jarnik [4], and a similar problem occurs in [7]. (For further information on number-theoretic methods, see [1] and its bibliography.) For $y=\left(1, y_{2}, \ldots, y_{n}\right)$ we denote by $T_{y}$ the orthogonal projection from $R^{n}$ onto the orthogonal complement of $y$. We shall show that

$$
\operatorname{dim}\left\{y: \operatorname{dim} T_{y}(E)<s\right\} \geq s,
$$

which is equivalent with

$$
\operatorname{dim}\left\{V \in G(n, n-1): \operatorname{dim} P_{V}(E)<s\right\} \geq s .
$$

Fix $\delta>0$ and let $A_{\delta}$ be the set of all $y=\left(1, y_{2}, \ldots, y_{n}\right)$ such that for arbitrarily large $k$ there is an integer $H$ for which $k<H<N_{k}^{1-\delta}$ and $\left\|H y_{j}\right\|<H N_{k}^{-n / s}$ for $2 \leq j \leq n$. Using [4, Satz 4] or [7], one finds that $\operatorname{dim} A_{\delta} \geq s(1-\delta)$. Since $s(1-\delta) \rightarrow s$ as $\delta \rightarrow 0$, it is sufficient to show that $\operatorname{dim} T_{y}(E)<s$ for $y \in A_{\delta}$.

Let $y \in A_{\delta}$ and let $k$ and $H$ be as above. Choose integers $b_{2}, \ldots, b_{n}$ such that $\left|H y_{j}-b_{j}\right|<H N_{k}^{-n / s}$ for $2 \leq j \leq n$ and denote $y^{*}=$ $\left(1, b_{2} H^{-1}, \ldots, b_{n} H^{-1}\right)$. Then $\left|y-y^{*}\right|<n N_{k}^{-n / s}$, whence $\left\|T_{y}-T_{y^{*}}\right\|<$ $O(1) N_{k}^{-n / s}$. $(O(1)$ stands for a constant independent of $k$.) Let $v=$ $\left(v_{1}, \ldots, v_{n}\right)$ with each $v_{j}$ an integer and $\left|v_{j}\right| \leq N_{k}$. We write $v_{1}=q_{1} H+r_{1}$, where $q_{1}$ and $r_{1}$ are the uniquely determined integers such that $0 \leq r_{1}<H$. Then

$$
v=\left(q_{1} H, q_{1} b_{2}, \ldots, q_{1} b_{n}\right)+\left(r_{1}, v_{2}-q_{1} b_{2}, \ldots, v_{n}-q_{1} b_{n}\right) .
$$

The first member on the right is parallel to $y^{*}$, while the second takes at most $H\left(3 N_{k}\right)^{n-1}=O(1) H N_{k}^{n-1}$ values, when $v$ varies. Hence also 
$T_{y^{*}}\left(N_{k}^{-1} v\right)$ assumes at most $O(1) H N_{k}^{n-1}$ values, and, by the definition of $E, T_{y^{*}}(E)$ can be covered by that many balls of radius less than $O(1) N_{k}^{-n / s}$. Inasmuch as $\left\|T_{y}-T_{y^{*}}\right\|<O(1) N_{k}^{-n / s}$, the same is true for $T_{y}(E)$. For $(n-\delta) s / n<\alpha<s, H N_{k}^{n-1}\left(N_{k}^{-n / s}\right)^{\alpha}<N_{k}^{n-\delta-n \alpha / s}$ tends to zero as $k \rightarrow \infty$; hence $\mathscr{H}^{\alpha}\left(T_{y}(E)\right)=0$. Therefore $\operatorname{dim} T_{y}(E)<s$, as required.

We next assume that $m<n-1$ and proceed by induction on $n$. So assume that there exists a compact set $E \subset R^{n-1}$ with $\operatorname{dim} E=s$ such that letting

$$
A=\left\{V \in G(n-1, m): \operatorname{dim} P_{V}(E)<s\right\},
$$

we have $\operatorname{dim} A=s+m(n-m-2)$. We identify $R^{n-1}$ with $\left\{\left(x_{1}, \ldots, x_{n}\right) \in R^{n}: x_{n}=0\right\}$. Let $G$ be the set of all $V \in G(n, m)$ such that $\left(x_{1}, \ldots, x_{n}\right) \in S^{n-1} \cap V$ implies $\left|x_{n}\right|<1 / 2$ and define $P: R^{n} \rightarrow R^{n-1}$ by $P\left(x_{1}, \ldots, x_{n}\right)=\left(x_{1}, \ldots, x_{n-1}\right)$ and $F: G \rightarrow G(n-1, m)$ by $F(V)=$ $P(V)$. Then $F$ is Lipschitzian (this can be seen with the help of 2.1) and $F^{-1}\{V\}$ is isometric with an open subset of $G(m+1, m)$ for $V \in G(n-1$, $m)$. Thus $\mathscr{H}^{m}\left(F^{-1}\{V\}\right)>0$ for $V \in G(n-1, m)$, and Lemma 6 implies that $\operatorname{dim} F^{-1}(A) \geq \operatorname{dim} A+m=s+m(n-m-1)$. Since $\operatorname{dim} P_{V}(E)<s$ for $V \in F^{-1}(A)$, we have shown that the set

$$
A_{1}=\left\{V \in G(n, m): \operatorname{dim} P_{V}(E)<s\right\}
$$

is of dimension $s+m(n-m-1)$.

To treat the case of $O^{*}(n, m)$, assume that $E$ and $A_{1}$ are as above and denote

$$
A_{2}=\left\{p \in O^{*}(n, m): \operatorname{dim} p(E)<s\right\} .
$$

Define $F: O^{*}(n, m) \rightarrow G(n, m)$ letting $F(p)$ be the orthogonal complement of $\operatorname{ker} p$. Then $F$ is Lipschitzian by 2.2 and $F^{-1}\{V\}$ is isometric with $O(m)$, whence $\mathscr{H}^{m(m-1) / 2}\left(F^{-1}\{V\}\right)>0$, for $V \in G(n, m)$. Moreover $A_{2} \supset F^{-1}\left(A_{1}\right)$. Using Lemma 6 we infer $\operatorname{dim} A_{2} \geq \operatorname{dim} F^{-1}\left(A_{1}\right) \geq$ $\operatorname{dim} A_{1}+m(m-1) / 2=s+m(2 n-m-3) / 2$. Thus $\operatorname{dim} A_{2}=$ $s+m(2 n-m-3) / 2$.

Finally we consider $\operatorname{Hom}\left(R^{n}, R^{m}\right)$ and set

$$
A_{3}=\left\{f \in \operatorname{Hom}\left(R^{n}, R^{m}\right): r(f)=m, \operatorname{dim} f(E)<s\right\} .
$$

Let $B$ be the set of all $f \in \operatorname{Hom}\left(R^{n}, R^{m}\right)$ such that $l(f)>1$ and $f=L \circ P_{V}$ for some $V \in G(n, m)$ and some non-singular linear mapping $L: V \rightarrow R^{m}$. Then $V$ is uniquely determined by $f$; in fact $V=(\operatorname{ker} f)^{\perp}$. We define $F: B \rightarrow G(n, m)$ setting $F(f)=V$ for $f=L \circ P_{V}$. Then $f$ is Lipschitzian by 2.2 and $F^{-1}\{V\}$ is isometric with an open subset of 
Hom $\left(R^{m}, R^{m}\right)$ for $V \in G(n, m)$, whence $\mathscr{H}^{m^{2}}\left(F^{-1}\{V\}\right)>0$. Clearly $A_{3} \supset F^{-1}\left(A_{1}\right)$. Therefore $\operatorname{dim} A_{3} \geq \operatorname{dim} F^{-1}\left(A_{1}\right) \geq \operatorname{dim} A_{1}+m^{2}=$ $s+m(n-1)$ and $\operatorname{dim} A_{3}=s+m(n-1)$.

\section{References}

[1] BAKer, A., and W. M. SchmidT: Diophantine approximation and Hausdorff dimension. - Proc. London Math. Soc. (3) 21, 1970, 1-11.

[2] EGgleston, H. G.: Sets of fractional dimension which occur in some problems of number theory. - Proc. London Math. Soc. (2) 54, 1952, 42-93.

[3] Federer, H.: Geometric Measure Theory. - Springer-Verlag, Berlin-Heidelberg-New York, 1969.

[4] JARNik, V.: Über die simultanen diophantischen Approximationen. - Math. Z. $33,1931,505-543$.

[5] Kaufman, R.: On Hausdorff dimension of projections. - Mathematika 15, $1968,153-155$.

[6] -»- An exceptional set for Hausdorff dimension. - Mathematika 16, 1969, $57-58$.

[7] -»- Probability, Hausdorff dimension and fractional distribution. Mathematika 17, 1970, 63-67.

[8] Matrila, P.: Hausdorff dimension, orthogonal projections and intersections with planes. - Ann. Acad. Sci. Fenn. Ser. A I 1, 1975, 227-244.

[9] Whitney, H.: Geometric Integration Theory. - Princeton University Press, Princeton, N. J., 1957.

University of Illinois

Department of Mathematics

Urbana, Illinois 61801

USA
University of Helsinki

Department of Mathematics

SF-00100 Helsinki 10

Finland

Received 25 August 1975 